FORMATLON Formation emploi

Revue française de sciences sociales

150 | avril-juin 2020

Former aux "petits" métiers : regards internationaux

\title{
Filles en lycée professionnel : quand la socialisation juvénile peut bousculer les socialisations, scolaire et professionnelle
}

Girls in vocationnal upper secondary school: socialisation in school and

professional socialisation

Mädchen in der Berufsschule : Wenn die Sozialisation der Jugendlichen mit der

Sozialisation in Schule und Beruf interferiert

Mujeres en la escuela secundaria técnica: cuando la socialización juvenil puede empujar las socializaciones escolar y profesional

\section{Séverine Depoilly}

\section{(2) OpenEdition}

\section{Journals}

Édition électronique

URL : https://journals.openedition.org/formationemploi/8154

DOI : 10.4000/formationemploi.8154

ISSN : 2107-0946

\section{Éditeur}

La Documentation française

Édition imprimée

Date de publication : 8 juillet 2020

Pagination : 79-96

ISSN : 0759-6340

Référence électronique

Séverine Depoilly, «Filles en lycée professionnel : quand la socialisation juvénile peut bousculer les socialisations, scolaire et professionnelle », Formation emploi [En ligne], 150 | avril-juin 2020, mis en ligne le 02 janvier 2022, consulté le 06 janvier 2022. URL : http://journals.openedition.org/ formationemploi/8154 ; DOI : https://doi.org/10.4000/formationemploi.8154 


\title{
Filles en lycée professionnel : quand la socialisation juvénile peut bousculer les socialisations scolaire et professionnelle
}

\author{
SÉVERINe Depoilly \\ Maîtresse de conférences en sociologie à l'Institut national supérieur du professorat et de \\ l'éducation de l'Université de Poitiers et chercheuse au laboratoire Gresco (Groupement de \\ recherches sociologiques sur les sociétés contemporaines)
}

Résumé

Filles en lycée professionnel : quand la socialisation juvénile peut bousculer les socialisations scolaire et professionnelle

À partir d'une enquête ethnographique au long cours conduite dans des lycées professionnels préparant à des domaines de spécialités variés, cet article interroge certains des principes socialisateurs, à la croisée des mondes scolaire et professionnel, qui gouvernent la formation des filles des milieux populaires. S'éclairent ainsi des mécanismes proprement scolaires qui participent à la reproduction de la division sociale et sexuelle du travail. Mais cet article se propose aussi d'interroger ce que les filles font de ce que l'on fait d'elles. Loin d'être les objets passifs de cette entreprise de façonnage de leurs corps et de leurs conduites, les filles se l'approprient et en font un usage propre en y opposant, en certains contextes et à certaines conditions, leur propre système de normes, de valeurs et de pratiques.

Mots clés : femme, lycée professionnel, division sexuelle du travail, BEP - brevet d'études professionnelles, baccalauréat professionnel, socialisation, socialisation professionnelle, identité sociale, genre, jeune

Abstract

Girls in vocationnal upper secondary school: socialisation in school and professional socialisation

Based on an ethnographic survey in vocationnal upper secondary school in different sectors of activity, this article analyses the socialisation of young girl. The socialisation in school reproduce a gender based division on labour. But the young girls are not passive, they can resist. In fact, some behavior may represent an active appropriation of school and professionnal socialisation. 
Keywords: woman, vocational upper secondary school, gender based division of labour, certificate of vocational education, vocational baccalaureate, socialisation, professional socialisation, social identity, gender, young person

Journal of Economic Literature: I 21, J 24

Traduction : auteure.

Le lycée professionnel (LP), parce qu'il noue ensemble socialisation scolaire et socialisation professionnelle et prépare le plus souvent aux métiers et aux diplômes relevant du travail d'exécution, est un espace singulier dans le paysage des institutions scolaires. Sa singularité tient aussi au public qu'il accueille. Il est scolairement et socialement relativement homogène. Les élèves de LP sont plutôt en difficulté scolaire, ont rarement fait le choix de leur orientation et sont le plus souvent issus des milieux populaires. Le lycée professionnel occupe ainsi une place "dominée » dans l'ordre des hiérarchies socio-scolaires (Grignon, 1971 ; Tanguy, 1983 ; Charlot, 1999 ; Jellab, 2001, Moreau 2006 ; Pahleta, 2012).

Le public scolaire des LP peut aussi souvent se caractériser par une non-mixité des sexes (Mosconi, 1987 ; Moreau, 1995 ; Mosconi \& Dahl-Lanotte, 2003 ; Lemarchant, 2017 ; Denave, 2019). Parce que le LP propose des voies de formation et prépare à des métiers souvent désignés, dans le sens commun, comme plutôt " masculins » ou "féminins », certaines de ses filières de spécialité peuvent être uniquement composées de filles ou de garçons. C'est notamment le cas des sections du service à la personne, de la coiffure, de l'esthétique, qui accueillent une très grande majorité de filles quand les filières du bâtiment ou de l'industrie restent quasi exclusivement composées de garçons. Dans ce paysage de l'enseignement professionnel, les filles occupent donc une place singulière, qu'elles se conforment aux attendus de leur sexe ou qu'elles décident de franchir les barrières de la sexuation des filières.

Cet article se propose de s'intéresser aux filles des milieux populaires scolarisées dans différentes filières du lycée professionnel. Un des objectifs de notre travail est de saisir certains des principes guidant le travail socialisateur qui s'opère en direction de ce public scolaire féminin. La variété des filières de formation envisagées dans le cadre de cette enquête permet en outre d'appréhender la permanence de certains des principes qui fondent les socialisations respectivement scolaire et professionnelle.

L'analyse de ces principes révèle qu'on attend de ces élèves qu'elles respectent une place relativement traditionnelle et dominée dans l'ordre de la division socio-sexuelle des tâches et du travail. Mais pour nous saisir pleinement de la densité de ce travail socialisateur (Darmon, 2006), nous nous intéresserons aussi à ce que les filles font de ce que l'on fait d'elles. À cet égard, l'analyse des formes d'entrecroisement, de juxtaposition ou de mise en concurrence des socialisations scolaire, professionnelle et juvénile, nous 
permettra d'éclairer les modes d'appropriation, par les filles, des injonctions scolaires et professionnelles dominantes.

En d'autres termes, il s'agira, d'une part, de repérer et d'analyser par quels biais et à partir de quels supports l'institution forme et transforme les filles qu'elle accueille (I) et, d'autre part, d'analyser, en contrepoint, la manière dont elles " s'arrangent " (Goffman, 1961) de ce travail socialisateur, s'y ajustent, s'en accommodent et/ou peut-être y résistent (Scott, 2008), bref, comment elles en font usage (II).

Nous préciserons d'abord deux points avant d'entrer plus avant dans l'analyse. Tout d'abord, notre choix de porter l'étude sur les filles ne signifie pas qu'elles seraient les seules destinataires de ce travail d'encadrement genré et classé de leurs comportements. Les garçons font eux aussi l'objet d'un travail similaire. Nous postulons d'ailleurs que c'est en réalité dans l'analyse relationnelle (Mathieu, 1971) de ces formes d'encadrement que nous pouvons repérer des répertoires de normes, de valeurs et de pratiques qui s'adressent plutôt aux filles ou plutôt aux garçons et qui dessinent de fait les contours d'un féminin ou d'un masculin populaire.

Ensuite, la réflexion que nous souhaitons engager ici vise à prendre un peu de distance avec une lecture et une interprétation des expériences scolaires des filles souvent univoques et injustement homogénéisantes, qu’il s'agisse de lire leurs comportements et attitudes scolaires sous le prisme de leur incorporation de dispositions à la docilité, à la soumission souvent transmises d'abord dans le cadre socialisateur familial (Baudelot, Establet, 1992) ou de les interpréter comme le résultat de leur adaptation raisonnable aux réalités de l'ordre social (Duru-Bellat, 2004). Ces approches tendent en effet à faire écran, d'une part, à la variété et la variabilité des expériences scolaires des filles ; d'autre part, au poids des différentes dynamiques socialisatrices qui prennent forme et contenu dans les quotidiens scolaires et qui laissent apparaître des appropriations tantôt partielles, tantôt hétérodoxes des attendus scolaires et professionnels sexués véhiculés et promus par le lycée professionnel. Ainsi, nous le verrons, les filles peuvent-elles contrevenir à ces attendus et ainsi revendiquer, dans l'espacetemps scolaire, l'existence d'un autre système de normes et d'une autre place dans ce système. 


\section{Encadré 1. Méthodologie : pratiques de terrain et logiques d'enquête}

Notre démonstration se fonde sur l'analyse de données d'enquêtes ethnographiques, conduites entre 2005 et 2018, dans cinq lycées professionnels situés, pour trois d'entre eux, en zones urbaines et pour les deux autres, en zones rurales. Ces enquêtes ont visé à mieux comprendre les expériences socialisatrices scolaires et professionnelles des élèves. L'investigation ethnographique, notons-le dès à présent, a visé une observation et une compréhension « dense » (1) (Lüdtke, 2015) de régularités dans l'intrication des réalités locales quotidiennes. Les filières de formation investies pour l'enquête sont variées. Du côté des filières communément désignées comme masculines, nous avons observé des classes de BEP (brevet d'études professionnelles (2) - Bac pro plomberie, maçonnerie, mécanique automobile, conduite poids lourds, pilote des systèmes automatisés et usinage ( 75 heures au total). Du côté des filières de formation traditionnellement désignées comme féminines, nous avons observé des classes de BEP coiffure, esthétique, des Bac pro soins et services à la personne (200 heures au total). Nous avons également observé des classes de BEP et de Bac pro relativement mixtes des secteurs de la vente, du commerce, et de la gestion administration (135 heures au total). L'observation de chacune de ces classes s'est associée à des enquêtes par entretiens (44 au total) conduits auprès d'élèves volontaires. Ces entretiens ont notamment permis d'aborder les thèmes suivants : la scolarité avant l'entrée en lycée professionnel, les choix d'orientation en classe de troisième, les vécus scolaires et de stage dans le cadre de la scolarité au lycée professionnel.

L'enquête ethnographique au long cours présente un avantage certain. Parce qu'elle se fonde sur une densification progressive du matériau d'enquête, elle permet le repérage de régularités au-delà même des filières de formation considérées et des configurations de classe qui y sont associées. Il ne s'agit pas ici de nier le possible effet des configurations de classe et des filières de formation sur le type de travail socialisateur engagé, mais notre propos vise plutôt, comme nous l'avons précédemment écrit, à faire état de ce qui apparaît comme transversal aux différents contextes de formation.

Au total donc, chacune de nos enquêtes nous a conduit à investir le terrain par observation à hauteur de 410 heures de présence au total dans les espaces classes, de vie scolaire et interstitiels (cour de récréation, cantine et couloirs). Nous avons interrogé une quarantaine d'élèves - filles et garçons - des différentes filières et avons échangé de manière plus informelle, mais aussi plus régulière, avec des enseignants et des CPE (conseiller principal d'éducation). Ces échanges informels ont ponctué les différentes vagues d'enquête. Ils ont surtout permis de saisir des représentations et de confirmer l'importance de points forts pour l'analyse. Chacun des établissements accueille une population scolaire relativement similaire d'un point de vue socio-scolaire : les élèves, filles et garçons, sont très largement issus des milieux populaires et de l'immigration pour deux des trois établissements situés en zone urbaine. L'orientation en lycée professionnel a le plus souvent relevé d'un second choix. 
Le travail d'analyse que nous livrons ici s'est construit sur la base d'un effort constant de mise en relation de données, souvent éparses. Ainsi, les matériaux, récoltés dans des contextes d'établissement différents, résonnent les uns avec les autres et permettent le repérage de lignes de force dans les processus socialisateurs en cours et leurs modes d'appropriation par les élèves. Pour autant, dans le cadre d'un article, il est délicat de rendre compte de la récurrence et des régularités observées, sur la base d'un matériau ethnographique. Les situations de classe que nous avons choisi d'exposer sont à considérer comme emblématiques de ce que les espaces de l'école ont permis d'observer afin de soutenir et d'argumenter notre propos. Les situations exposées et les extraits d'entretiens mobilisés ne sont pas isolés, mais représentent une "série homogène de cas observés » (Lahire, 1993).

(1) Cela suppose d'accorder une attention particulière, d'une part, au point de vue des enquêtés engagés dans les situations observées et, d'autre part, aux régularités observables dans l'intrication des réalités locales quotidiennes.

(2) Nous gardons les terminologies en usage au moment de l'enquête. Pour les classes de maçonnerie, de plomberie, d'esthétique et de coiffure, nous avons enquêté avant la mise en oeuvre de la réforme du Bac pro 3 ans, donc dans des classes de BEP.

\section{Pour les mondes scolaire et professionnel : un féminin populaire respectable}

Quelles que soient les configurations scolaires dans lesquelles elles se trouvent (classes mixtes, majoritairement masculines ou féminines), les filles de milieux populaires font l'objet d'un travail d'encadrement serré de leur corps et de leurs conduites qui est d'autant plus puissant qu'il prend pour appui et justification les modes de fonctionnement du monde professionnel. La socialisation opère ainsi de manière diffuse, par le langage et les discours des agents scolaires, et s'organise autour de deux principes : contrôler son apparence et savoir rester à sa place.

\subsection{Contrôler son apparence}

Dans les lycées professionnels enquêtés, sous des formes et avec des degrés d'intensité variables, les filles sont régulièrement rappelées à l'ordre du nécessaire contrôle de leur corps et de leur apparence qui doivent être ajustés aux univers professionnels envisagés. L'encadrement des modes de présentation et d'esthétisation de soi va ainsi constituer un premier mode de socialisation professionnelle.

"Tenue correcte adaptée aux besoins de l'entreprise ", "élégance », " sobriété », " hygiène » sont autant des termes fréquemment mobilisés par les enseignants des disciplines professionnelles des filières du secteur tertiaire. Il s'agit d'expliciter notamment, mais pas exclusivement, aux jeunes filles, ce qu'on attend d'elles dans le cadre professionnel. Les dispositions corporelles, la bonne esthétisation et le soin de soi qu'elles vont devoir 
apprendre à mobiliser sont présentés comme des compétences professionnelles centrales et indispensables pour qui prétend être au contact quotidien d'une clientèle ou d'usagers. Les moments de préparation au stage en milieu professionnel ou les journées " tenues professionnelles ", régulièrement organisées dans les filières de la vente et de la gestionadministration pour préparer leurs élèves à l'entrée dans la vie professionnelle, sont autant d'occasions, pour les enseignants, de travailler à l'inculcation des bonnes dispositions.

Enquêtrice : "Tu donnes des consignes précises à tes élèves avant qu'ils partent en stage ou pour les journées Tenue professionnelle?"

Salima : "Ah oui oui, je suis obligée, sinon ils font pas et ils comprennent pas toujours, mais je leur dis, c'est très simple: "restez sobre, ni trop court, ni trop décolleté". Ils ont tendance à confondre tenue professionnelle et "fashion victim". Une fois, une gamine, elle est entrée en cours, elle sétait maquillée pour la journée tenue professionnelle, mais un pot de peinture. J'ai

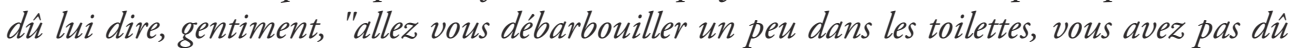
bien vous regarder ce matin" "(Salima, 38 ans, enseignante de vente en Bac pro commerce depuis 15 ans).

Le contrôle de l'apparence signifie ici que l'élève est capable de prendre de la distance à l'endroit des codes et des attitudes vestimentaires qui ont cours dans l'ordre de la sociabilité juvénile. Il s'agit bel et bien, pour ces jeunes filles, de montrer qu'elles entament un travail de transformation de certaines de leurs dispositions afin de les rendre plus conformes aux règles qui organisent les univers professionnels.

Cette transformation suppose une distanciation d'avec une féminité adolescente populaire ici désignée, par l'enseignante, par la figure de la fashion victim, fondée sur l'attachement des jeunes filles aux vêtements à la mode et, préférentiellement, de marque, calquée sur les pratiques vestimentaires et esthétiques des stars du moment et jugée peu conforme à l'élégance, la sobriété et la bonne tenue attendues dans le cadre professionnel. Les filles devraient ainsi pouvoir montrer qu'elles se démarquent du style des jeunesses populaires largement valorisées dans l'entre-soi adolescent (Mardon \& Zeroulou, 2015 ; Court, 2010) et qu'elles sont capables d'affirmer une féminité justement dosée et qui franchit les barrières de l'âge : la fashion victim adolescente doit pouvoir se transformer en jeune adulte respectable. Le travail du corps, de l'apparence, l'esthétisation de soi supposent d'être féminine, mais pas vulgaire, menace qui semble constamment pesée sur ces jeunes filles des milieux populaires, et conjurée par le conseil immuable du «ni trop court, ni trop décolleté ».

Le contrôle de l'apparence va plus loin qu'une intervention sur les corps et l'apparence. Il se trouve aussi possiblement associé, selon les filières considérées, à une forme d'encadrement moral des jeunes filles. Ce dernier est particulièrement fort dans les filières du soin à la personne ; les filles étant rappelées à la nécessaire bonne tenue de leur corps, gage de leur adaptation aux univers professionnels. C'est dans cette perspective que les tenues et les apparences corporelles sont rigoureusement encadrées. 
Avant chaque entrée en salle santé, les jeunes filles sont "inspectées ", les enseignant·e.s vérifient qu'elles ont les ongles coupés et non vernis, les cheveux attachés, qu'elles ne portent ni piercings, ni maquillage. L'argument principal avancé par les enseignants est celui de faire intégrer l'importance du respect des règles d'hygiène et de sécurité drastiques qu'elles auront à respecter dans leurs futurs lieux d'exercice professionnel. Mais le respect de la bonne tenue professionnelle va plus loin ; il doit aussi venir manifester la décence de ces jeunes filles destinées à entrer en contact régulier avec des usagers dont elles devront prendre soin.

Alicia : "Une fois je suis venue à la journée déguisée, habillée comme elles, mais genre jean super moulant, débardeur, on voit les seins, pantalons, on voit le string, super maquillage. Elles étaient choquées. Après je leur ai dit, "mais vous voyez, ça vous plairait vous si vous êtes patient dans une structure, une professionnelle qui se balade comme ça?" "(Alicia, 32 ans, enseignante en économie sociale et familiale en Bac pro soins et services à la personne depuis dix ans).

Lisa : "Parfois, sous leur blouse, on voit tout au travers, je leur dis de faire attention niveau sous-vêtements, que ça reste correct et propre. " (Lina, 28 ans, enseignante d'économie sociale et familiale en Bac pro soins et services à la personne depuis six ans).

Les attitudes corporelles et les pratiques vestimentaires des jeunes filles sont ici tournées en dérision par Alicia, pour mieux en montrer le caractère inadapté dans le cadre de la pratique professionnelle. Les jeunes filles doivent ici prendre la mesure du manque de décence sous-tendue par un corps jugé trop dévoilé ou sexualisé. Ceci étant susceptible de mettre en doute la possession, par les jeunes filles, des bonnes dispositions morales qui sont là aussi précisément énoncées par les enseignants : capacité d'écoute, empathie, dévouement, disponibilité, amabilité, sympathie, discrétion, réserve, autant de qualités traditionnellement associées au féminin. L'hexis corporelle, c'est-à-dire l'ensemble des manières d'être, d'agir, de se tenir, qu'il s'agit de transmettre aux jeunes filles dans ce cadre scolaire professionnalisant, dessine bel et bien les traits d'un certain féminin populaire acceptable et respectable (Skeggs, 2015) : une féminité mesurée et contenue qui doit tenir ses distances d'avec une féminité adolescente et populaire jugée potentiellement vulgaire et outrancière.

\subsection{Savoir rester à sa place}

Quels que soient les champs de formation considérés, même si les formes et les degrés d'intensité peuvent varier en fonction notamment de la formation suivie et des configurations de classes qui y sont associées, les filles sont rappelées à l'ordre de la nécessité de savoir rester à leur place, notamment dans la hiérarchie de la division sociale et sexuelle des tâches et du travail. Savoir occuper et garder cette juste place s'opère de deux manières différentes : par un processus de délégitimation-dépréciation du féminin, d'une part, et par un processus de déresponsabilisation-naturalisation des garçons, d'autre part. 
S’observent ainsi différentes formes de dépréciation-délégitimation du féminin qui peuvent être portées par des garçons, des agents scolaires ou les filles elles-mêmes. Les filles sont ainsi fréquemment réduites à leur corps, le corps-objet devenant le principal support de leur désignation et de leur détermination dans l'espace de la classe.

Nous nous référerons ici au cas de Marlène, seule fille parmi quinze garçons de sa classe de terminale Bac pro, pilote de ligne de production au moment où nous la rencontrons. Marlène rapporte, en entretien, des propos qui ont été tenus par un enseignant d'atelier lors de son année de seconde Bac pro.

Marlène : "Et en fait, j'avais un prof misogyne (l'enseignant dont parle Marlène est un homme, 55 ans, professeur d'atelier depuis 20 ans) très très souvent et qui me faisait des remarques, par exemple sur mon tee-shirt des choses comme ça. " Enquêtrice : "Et vous avez des souvenirs précis de ce qu'il pouvait vous dire? Marlène : "Ah ben, Marlène elle a mis son décolleté pour avoir une bonne note, alors que pour moi, c'est un tee-shirt basique ... . Et en fait, j’ai pété un plomb avec les garçons parce que, eux, forcément, ça les faisait rire et c'était très épuisant pour moi " (Entretien avec Marlène, 18 ans, Terminale Bac pro pilote de ligne de production).

Marlène est ici réduite à ses atours esthétiques, à la nature de son " être-femme ", qui servent à la délégitimer du point de vue scolaire et technique et à la tenir à distance du collectif masculin. En soupçonnant Marlène d'user de son corps pour obtenir une reconnaissance de ses capacités scolaires et techniques matérialisées par la bonne note, on la prive de la possibilité d'apporter la preuve de sa maîtrise de certaines compétences. On lui signifie en outre qu'elle n'est, par conséquent, pas à sa place. Devenue objet d'amusement pour les garçons, elle est progressivement exclue du collectif masculin et donc du collectif de la classe.

On peut rapprocher cet événement énoncé par Marlène des témoignages de mises à l'épreuve - et dont la littérature scientifique (Mosconi, op. cit. ; Mosconi \& DahlLanotte, $o p$. cit. ; Lemarchant, $o p$. cit.) a pu déjà largement rendre compte - que nous avons pu recueillir auprès de jeunes filles scolarisées dans des filières majoritairement masculines. De fait, le contenu même des propos de l'enseignant tient très certainement en partie à la configuration singulière dans laquelle se trouve Marlène. Marlène est la seule fille d'une classe de quinze garçons et son professeur d'atelier est un homme d'âge mûr qui a fait une longue carrière en usine avant d'intégrer l'enseignement. Pour autant, on trouve ici à l'œuvre, non pas un événement isolé dépendant uniquement d'une seule configuration mais, sous la forme d'un miroir grossissant, un mécanisme relativement fréquent d'exclusion des filles de la compétition scolaire, comme l'illustre, autrement, la situation ci-dessous.

Les élèves d'une classe de Bac pro gestion administration doivent corriger au tableau des exercices de mathématiques. Salim se propose de corriger le premier exercice. Il se trompe dans ses lignes de calcul. L'enseignante demande aux autres élèves de l'aider. 
Shaïma intervient : "Non mais, tu t’es trompé là dans ton calcul. C'est pas la bonne ligne" Salim : "Putain ta gueule, reste à ta place toi, tais-toi va!"

L'enseignante : "Oh, calmez-vous »

Salim : "Ouais, mais pourquoi elle parle, elle?".

Shaïma souffle pour montrer son exaspération, puis poursuit ses calculs (Observation en Bac pro vente, cours de mathématiques, janvier 2014).

Exclure les filles du travail sur machine ou avec les outils, leur imposer des mises à l'épreuve visant à leur rappeler le désajustement de leur corps au travail physique, les priver de la possibilité de prendre la parole dans l'espace classe, les provoquer en usant d'insultes ou en faisant des blagues à caractères sexuels ou pornographiques sont autant de moyens mobilisés pour les rappeler à l'ordre de la division sociale et sexuelle des tâches et à leur subordination. Les filles apprennent ainsi à sortir du jeu de la compétition, à ne pas concurrencer les garçons, bref, à ne pas contester le monopole des hommes sur certains instruments, savoirs et domaines de pratiques (Tabet, 1979), à rester peu visibles et relativement silencieuses.

Il n'est pas rare que les jeunes filles soient rendues responsables des débordements et des provocations des garçons dont les filles se disent victimes. Le pendant de cette responsabilisation des filles est en l'occurrence la déresponsabilisation des garçons, renvoyés, eux-aussi, à leur nature d'homme en devenir, à leur supposée immaturité physiologique (Collet, 2014), facteur explicatif central de leurs attitudes. Parce que responsables, les filles sont donc invitées à réguler leurs attitudes, notamment corporelles et vestimentaires qui, souvent jugées par les agents scolaires comme trop sexualisées et donc comme potentiellement provocatrices, doivent manifester leur vertu et donc, leur respectabilité sexuelle. Mais les jeunes filles semblent devoir apprendre davantage encore : elles doivent apprendre à faire avec la nature des garçons. Enfermer les garçons dans leur supposée nature immature, c'est aussi enfermer les jeunes filles dans une position de nécessaire soumission à ces mêmes faits de nature sur lesquels, par définition, il est impossible d'agir. Ainsi en est-il de Léane, qui rencontre des difficultés d'intégration dans sa classe de maintenance des véhicules automobiles et qui est reçue en entretien par son professeur principal, professeur d'atelier de mécanique auto, et le CPE :

Léane (16 ans, Seconde Bac pro maintenance des véhicules, voitures particulières) se plaint de l'attitude des garçons de sa classe qui l'empêchent, selon elle, de se mettre au travail sérieusement.

Léane : "Non mais, vous voyez comment ils me parlent, ils me prennent pour un mec alors que je suis une fille. J'aime pas qu'on me parle mal." 
Professeur principal (48 ans, un homme enseignant de mécanique automobile depuis 23 ans) : "Mais tu fais toujours des remarques, tu finis par les agacer les garçons, avec ton côté moralisatrice, tu n'es pas un peu sèche parfois avec eux?"

Léane : "Non, mais moi je voudrais changer de groupe."

CPE (un homme, 40 ans, CPE depuis 15 ans en lycée professionnel) : "C'est vrai que tu n'as pas de chance et tu en as peut-être trop accepté. L'année prochaine, ça ira mieux, ils auront un an de plus, ce sera plus facile, ils seront plus matures. Le problème, c'est que tu es plus mûre qu'eux."

Professeur principal : "Et puis quand même, tu n'es pas tombée dans la classe la plus difficile."

Léane: "De toute façon, $j$ 'aurais un caractère moins fort, j'y arriverai plus ici» (Observation vie scolaire, novembre 2016).

Ce qui caractérise le plus cet échange, c'est très probablement la mise en cause des modes de comportement de Léane face aux garçons, comportements jugés inadaptés au contexte de scolarisation qui est le sien, un contexte très majoritairement masculin. Successivement soupçonnée d'être agaçante, trop moralisatrice, trop sèche ou d'en avoir trop accepté, Léane est finalement bel et bien renvoyée à son incapacité à trouver sa juste place. Cela suppose en réalité d'accepter et de respecter la frontière fondée sur un principe de naturalisation de la différence entre masculin et féminin, garçons et filles.

Or, dans cet extrait, Léane revendique son droit à être reconnue comme sujet femme. Aux différences de maturité qui sont invoquées comme mode d'explication à ses difficultés d'intégration, elle répond en réalité par son non-respect de la frontière qui sépare un masculin dominant d'un féminin nécessairement dominé. En répondant aux différences de nature par la mobilisation du prisme explicatif du trop fort caractère, Léane outrepasse ses prérogatives, transgresse les frontières, moins en refusant le principe de différenciation, qu'elle peut d'ailleurs mobiliser pour elle-même en revendiquant sa position de "fille ", qu'en contestant les principes d'asymétrie et de hiérarchisation qui lui sont associés.

Ces mécanismes visent en l'occurrence à rappeler aux jeunes filles qu'elles se doivent finalement de respecter les principes d'asymétrie, de hiérarchie et de différenciation qui organisent les rapports sociaux de sexe dans des environnements scolaires, puis professionnels. Ceux-ci, malgré leurs configurations variées, supposent que les jeunes filles ne déstabilisent ou ne gomment pas les frontières qui les séparent du masculin.

Les expériences scolaires des filles se trouvent ainsi enserrées, dans une certaine mesure, dans des rapports sociaux de domination de sexe et de classe. Elles font régulièrement l'objet d'une mise en conformité genrée et classée (Skeggs, op. cit. ; Kergoat, 2014 ; Kergoat, 2018 ; Chaponnière, Fassa, Lamamra, 2014) qui préfigure celle qui traver- 
sera, quotidiennement, leurs autres expériences sociales, et notamment leurs expériences au travail. Notre matériau invite néanmoins à la prudence ou à tout le moins à la nuance. La permanence de certains mécanismes n'exclut pas que les filles puissent résister à ces injonctions à être et à faire certaines choses. C'est ce que nous nous proposons d'examiner maintenant.

\section{Des dispositions juvéniles pour contrer l'emprise scolaire et professionnelle}

Pour nous saisir des formes d' " arrangement " (Goffman, 1961) avec les normes de genre classées mobilisées par les jeunes filles observées, nous porterons attention aux différentes logiques socialisatrices à l'œuvre dans l'école et les classes : les logiques scolaires, professionnelles et juvéniles. La façon dont les logiques socialisatrices à l'œuvre vont trouver à se combiner, coexister ou à se concurrencer manifeste une appropriation hétérodoxe des normes scolaires et professionnelles dominantes.

Dans les lignes suivantes, nous nous intéresserons à certaines des logiques socialisatrices féminines juvéniles et certaines des dispositions ${ }^{1}$ qui leur sont associées. Il s'agit de montrer qu'elles peuvent constituer un support pour mettre à distance et contrer certaines des assignations identitaires genrées et classées adressées aux filles de LP.

Précisons cependant que l'attention accordée à la socialisation juvénile ici tient au matériau dont nous disposons. Nous ne pensons pas qu'il s'agisse là du seul support de socialisation mobilisable pour les filles. En l'occurrence, les socialisations familiales comme scolaires, notamment incarnées dans certaines relations aux adultes et notamment aux enseignants (Kergoat \& Jarty, 2017 ; Jellab, 2005), peuvent, à certaines conditions, et en certains contextes, permettre aux filles d'opposer d'autres modèles.

\subsection{S'affirmer adolescente et contrer l'injonction à la responsabilité}

Les entretiens conduits avec les jeunes filles permettent de repérer une revendication récurrente, celle d'avoir le droit de rester jeune : "faut quand même qu'on profite, on est jeunes", "moi je veux pas travailler tout de suite, je veux profiter", "nous dans notre tête, on veut encore s'amuser, c'est normal ». Mais la revendication à profiter de sa jeunesse tend à entrer en contradiction avec ce que le lycée professionnel propose aux élèves, tant en termes de contenus que de modalités de formation, et qui les enjoint de franchir, plus vite que les autres élèves des filières générales, les barrières de l'âge. Ainsi, aux injonc-

1. Il ne s'agit pas pour nous ici de postuler que l'observable, chez les filles, relèverait de dispositions plutôt ou exclusivement "féminines ". En l'espèce, certaines des manières d'être et de faire, mobilisées par les filles, sont très proches de ce que l'on a pu observer au sein des groupes de pairs masculins. 
tions à la responsabilité portée par les enseignants, ces derniers prenant appui sur les contextes professionnels qui imposent des relations entre adultes et non entre jeunes, les filles répondent par leur droit à profiter de leur jeunesse.

Cet état de jeunesse se repère en différents endroits du lycée professionnel. De manière manifeste, dans les espaces dédiés à la sociabilité juvénile (cour de récréation, cantine, espaces interstitiels et abords proches de l'établissement) et de manière plus ou moins visible et licite dans les salles de classe. Ainsi, certains des usages des corps, des gestes, des voix, du langage peuvent laisser apparaître des filles particulièrement "indociles" (Kergoat, 2018) et de fait, relativement peu perméables aux normes de genre classées, telles que les véhiculent les mondes scolaires et professionnels.

L'usage illicite de téléphones portables, les postures manifestant l'endormissement ou la lassitude, la lecture de magazines ou de livres, les échanges entre élèves plus ou moins discrets, l'usage du maquillage, les séances de coiffage, le grignotage de chips ou de bonbons, les diverses formes de cajoleries entre filles, les interpellations entre élèves ou celles de l'enseignant, plus ou moins conformes et en rapport avec les attendus scolaires, les digressions très fréquentes que peut susciter tel propos de l'enseignant, les départs précipités de la salle de classe quelques secondes seulement avant la sonnerie de fin des cours sont autant d'indices qui manifestent, de la part de ces jeunes filles, des formes d'appropriation singulière de l'espace scolaire.

Si elles peuvent ne jamais se positionner contre le travail de l'enseignant (Depoilly, 2012) ou l'activité d'apprentissage et montrer d'ailleurs un certain type d'intérêt - en demandant par exemple à lire les textes sans répondre aux questions, en faisant l'exercice demandé tout en poursuivant les discussions dans l'entre soi-juvénile - bref, en s'investissant en pointillé et dans des formes supposant peu de contraintes des corps, des voix et du langage -, les filles manifestent une prise de distance par rapporrt aux attendus scolaires traditionnels.

Ainsi n'est-il pas rare que les filles mobilisent des dispositions propres à l'entre-soi juvénile dans la classe et dans le temps des apprentissages. Les allusions, en présence des agents scolaires, à leurs vies festives, supposant la consommation immodérée d'alcool ou de stupéfiants, les interpellations relatives aux vies amoureuses ou sexuelles, les pratiques de la vanne, les usages de l'insulte, les interpellations grossières et sexualisées conduisent les filles à opposer aux règles et normes scolaires et professionnelles, leur propre système de dispositions construit dans l'entre-soi juvénile.

Diane, une élève de Bac pro, vient de donner une bonne réponse à la question posée par l'enseignante.

Anaëlle l'interpelle en riant : "Et Diane, tu suces ou quoi?"

L'enseignante (28 ans, enseignante depuis quatre ans) : "Non mais les filles, c'est pas croyable, y'a pas plus vulgaire qu'Anaëlle en fait. " 
Anaëlle et sa voisine de table rient. Anaëlle répond : "Ah mais non Madame, c'est rien, c'est pas grave, on a rien dit là. " (Observation de classe $1^{\text {ìre }}$ Bac pro soins et service à la personne, cours d'économie sociale et familiale).

En empruntant au vocabulaire sexuel, que les enseignants tendent par ailleurs à associer davantage aux garçons et donc à mieux tolérer chez ces derniers, les filles transgressent les normes de genre et scolaires qui leur imposent retenue et discrétion. En usant de l'interpellation sexualisée, elles ne visent pas d'abord la déstabilisation provocatrice et ostentatoire du travail de l'enseignant (Depoilly, op. cit. ; 2014). Elles semblent plutôt jouir du simple plaisir de rire, de s'amuser et d'entretenir des liens de camaraderie (Clair, 2017), dont l'enseignant n'est d'ailleurs pas nécessairement exclu, mais auquel elles opposent leur insouciance.

Il s'agit moins ici, pour les filles, de nier qu'elles ont pu être vulgaires que de dédramatiser cette vulgarité en la replaçant dans l'ordre des normes et des pratiques juvéniles. C'est ainsi à l'injonction à se montrer raisonnable et adulte que les filles résistent.

\subsection{Prouver sa force de caractère et contester l'asymétrie}

Prouver qu'on a un " caractère fort » est une des autres modalités permettant aux filles de mettre à distance certaines normes de genre et les rapports de sexe qui y sont associées. "Avoir un fort caractère " est un véritable fait de langage ; il apparaît de manière récurrente dans les entretiens conduits avec les filles, ses formes de mise en œuvre sont multiples et dépendantes des situations et des contextes. Il peut se manifester tant dans un face-à-face avec un pair du même sexe ou du sexe opposé qu'avec un adulte.

Le fort caractère ne se limite ni à la masculinisation, virilisation des attitudes des jeunes filles enquêtées (Rubi, 2005, 2018 ; Beunardeau, 2014) ni à leur transexualisation, dont la figure du "garçon manqué " serait la manifestation première, pas plus qu'il n'est réductible à une affirmation de soi par l'affrontement ou la déviance dans le cadre scolaire. Les entretiens et les observations de classe nous invitent à penser que les jeunes filles peuvent apporter la preuve de leur force de caractère de deux manières différentes au moins et non exclusives l'une de l'autre. Nous illustrerons chacune d'elles successivement.

Le fort caractère, c'est pouvoir montrer qu'on n'a pas peur, qu'il s'agisse de se défendre verbalement ou physiquement en cas de provocation ou de dire les choses franchement, en face-à-face, quitte à ce que cela nécessite des mises au point parfois houleuses. Elles peuvent adopter une attitude frontale en provoquant ou en répondant au « coup pour coup " aux rebuffades, moqueries ou insultes, notamment dans les relations avec les pairs, filles ou garçons. Notons que ces modes de faire ne s'exercent pas exclusivement au cours d'interactions qui engageraient filles et garçons; ils peuvent aussi être mobilisés face à des enseignants ou d'autres filles, l'enjeu étant de montrer qu'on ne se laisse 
pas faire. Examinons la manière dont Jenny répond à la provocation de Touffik dans la scène ci-dessous :

Avant d'entrer dans la salle de cours, dans les escaliers, Touffik bouscule très vivement Jennifer qui me tombe dessus. En guise de réponse, Jennifer hurle sur Touffik de manière particulièrement agressive :

"Mais t'es trop con, tu sais pas quoi faire ou quoi, dégage va et regarde comment t'es habillé, t'as l'air d'un clown, sale con va."

Touffik s'éloigne en riant.

Si la provocation de Touffik se situe peut-être dans l'ordre de l'amusement et de la plaisanterie, Jenny ne va pas hésiter à manifester son agacement. Son emportement verbal vient précisément manifester qu'elle prend toutes ses distances avec certains des attendus souvent formulés à l'endroit des jeunes filles : qu'elles acceptent l'immaturité constitutive des garçons contre laquelle il serait impossible de lutter. Dans son mode de réponse à Touffik, elle procède d'ailleurs à une inversion de la hiérarchie entre masculin et féminin. Elle le ridiculise en prenant notamment appui sur ses attributs vestimentaires pour le décrédibiliser en l'enfermant dans les figures du "clown» et du « sale con». Notons que les figures du "con», "p'tit' con", "sale con ", du " clown " ou du " gamin " sont très fréquemment mobilisées par les jeunes filles. Elles manifestent, comme celle du « macho ", sur un autre registre, le désajustement de ces garçons à l'endroit d'un masculin acceptable, soit que leur soit reproché des excès de virilité, comme tend à l'incarner la figure du « macho », soit qu'on leur signifie qu'ils n'ont précisément pas encore atteint l'âge d'homme.

Mais les filles peuvent aussi apporter la preuve de leur force de caractère en montrant qu'elles savent encaisser les coups durs, les vexations, les mises à l'épreuve possiblement imposées par les adultes dans les situations scolaires ou de stage.

Émeline est en classe de $1^{\text {ère }}$ Bac pro usinage. Elle nous fait état, en entretien, des formes de mises à l'écart dont elle a fait l'objet, notamment dans le cadre de ses stages.

Enquêtrice : "Comment ça s'est passé ta première période de stage?"

Émeline : "Ben parfois, certains sont machos, surtout les personnes âgées, enfin, les plus vieux. C'est plus compliqué. Mon premier stage, je suis arrivée, il y a un employé qui ma pas parlé pendant une semaine parce que jétais une fille. "

Enquêtrice : "Ahoui?"

Émeline : "Le patron il était gentil, mais l'employé, j'ai eu vraiment du mal avec lui vu que c'était vraiment "attends, je porte ça, c'est trop lourd, t'es une fille, les filles ça a pas de force". J'ai quand même réussi, parce que je suis quelqu'un de très têtue, si on me dit "ty arriveras pas", quitte à me faire mal, je le ferai quand même. "

Émeline est exclue des tâches dont on considère qu'elles sont inadaptées aux attributs de son sexe. Elle fait ainsi l'objet d'une forme de sexisme par paternalisme face auquel elle n'est 
finalement pas sans réponse. Catégorisant les hommes auxquels elle a affaire du côté de ces hommes d'âge mûr qui n'ont pas su évoluer avec leur temps et leur société, les inscrivant dans un autre temps, une autre génération à laquelle elle n'appartient pas et dont elle se distingue, elle se positionne en affirmant sa distance à l'égard de ces derniers, en même temps que sa singularité, "je suis quelqu'un de très têtue ", et ainsi un certain pouvoir d'agir.

De ce point de vue, elle défie la place qui lui est imposée et symbolisée par la phrase sanction " t'y arriveras pas", et apporte la preuve de sa capacité à faire le métier en manifestant notamment sa résistance à la douleur. Tout en empiétant sur le terrain des dispositions traditionnellement qualifiées de masculines, elle conteste la position de subordination dans laquelle l'environnement professionnel fréquenté tend à vouloir l'installer. Émeline ne se distingue donc pas de nombre de jeunes filles qui investissent les bastions masculins et imposent leur présence en résistant aux mises à l'écart dont elles peuvent faire l'objet. Ainsi transgressent-elles les frontières traditionnelles du genre.

On pourrait certainement interpréter chacune de ces situations tout autrement. On pourrait par exemple considérer que Jenny met en ouvre des traits de comportement plutôt traditionnellement désignés comme masculins. On pourrait de même lire l'entêtement d'Émeline comme la simple manifestation de dispositions à la docilité. Mais les penser ainsi, c'est s'empêcher de considérer le sens que ces modes de comportement prennent pour les filles elles-mêmes. C'est de fait nier leur capacité à mettre bel et bien en œuvre des manières d'être et de faire qui bousculent et déstabilisent les frontières qui séparent traditionnellement masculin et féminin. En prêtant attention, d'une part, à ce que les filles font effectivement en classe, dans la variété des situations considérées et, d'autre part, en écoutant ce qu'elles disent et ce qu'elles font, le chercheur peut prétendre pouvoir accéder à la complexité des processus qui font les expériences ordinaires de ces filles des milieux populaires.

Pour autant, d'un point de vue interprétatif, il convient de rester prudent. Il serait certainement abusif d'associer les agissements des filles ici décrits comme des ripostes collectives et conscientes contre des situations de domination scolaire et/ou de domination masculine, mais il le serait sûrement tout autant de ne les penser que comme "insoumission discrète " (Pahleta, op. cit. ; Marry, 2014). L'analyse des pratiques et des comportements mobilisés par les filles dans le cadre scolaire ne se laisse pas enfermer dans la bipolarité de "l'obéissance ou de la résistance " (Comaroff, 1985 ; Lüdtke, op. cit.). L'observation régulière des espaces scolaires laisse plutôt penser qu'il s'agit là d'une manière d'être au scolaire, qui s'est construite sur le long cours d'une trajectoire marquée par les difficultés, les échecs et la disqualification, et qui permet de se créer des espaces d'autonomie. Cette manière d'être au scolaire est le plus souvent tolérée par des enseignants qui se disent conscients de la particularité de leur public d'élèves (Jellab, 2005 ; Kergoat \& Jarty, op. cit.). 


\section{Conclusion}

Le lycée professionnel vise la transmission d'un certain nombre d'attitudes, de manières d'être et de faire jugées nécessaires à la réussite du parcours de formation et à une bonne insertion sur le marché du travail. Le répertoire des normes imposé aux jeunes filles de l'enseignement professionnel, s'il s'applique avec des degrés d'intensité variables selon les filières de formation considérées, exige qu'elles puissent mobiliser d'autres manières d'être, de faire et d'agir.

Elles sont simultanément invitées à l'effacement, à la responsabilité et font l'expérience de la dissymétrie et de la hiérarchisation dans les rapports sociaux d'âge, de sexe et de classe sociale. Le lycée professionnel préfigure ainsi la division socio-sexuée des tâches et du travail à laquelle les filles sont finalement préparées.

Mais à ce répertoire de normes, de valeurs, de pratiques fortement genrées et classées, les filles peuvent opposer un autre système dispositionnel, celui qui a trait aux logiques socialisatrices juvéniles et dont certaines des composantes peuvent emprunter, sans s'y réduire, au registre des normes, valeurs et pratiques qu'on associe plus traditionnellement au masculin populaire. En revendiquant leur droit à profiter de leur jeunesse, en s'appropriant les espaces scolaires et les activités d'apprentissage, en contestant certaines des frontières du genre qui les infériorisent, les jeunes filles bousculent les rapports d'âge, de sexe et de classe dans lesquels on les enserre.

Bien sûr, toutes les filles n'opposent pas, invariablement et toujours avec la même intensité, leurs logiques socialisatrices juvéniles aux logiques socialisatrices respectivement scolaire et professionnelle. Des données de contexte et de configurations de classe doivent nécessairement être prises en compte afin de comprendre pourquoi, comment, à quelles conditions et jusqu'où les filles peuvent mettre à distance les injonctions scolaires et professionnelles fortement genrées et classées auxquelles elles ont à faire face ; cependant, les manières variées de s'arranger avec le (et $\mathrm{du})$ monde scolaire manifestent, sans que nous ne cédions ici à une vision romantique du pouvoir d'opposition et de contestation des filles, des formes d'appropriation des quotidiens scolaires qui ont leur logique propre. Il convient de les prendre au sérieux.

\section{Bibliographie}

Baudelot C., Establet R. (1992), Allez les filles!, Paris, Seuil.

Beunardeau P. (2014), "Filles et conduites 'viriles'. L'identité féminine réinventée des 'Niafou' ", in Pour en finir avec la fabrique des garçons, dir. Ayral S., Raybaud Y., Pessac, MSHA.

Chaponnière M., Fassa F., Lamamra N. (2014), «Formation professionnelle. L'apprentissage des normes de genre ", Nouvelles questions féministes. 
Charlot B., Figeat M. (1985), Histoire de la formation des ouvriers, 1789-1964, Paris, Minerve.

Charlot B. (1999), Le rapport au savoir en milieu populaire. Une enquête dans les lycées professionnels de banlieue, Paris, Anthropos.

Clair I. (2017), " S’insulter entre filles. Ethnographie d'une pratique polysémique en milieu populaire et rural ", Terrains et travaux, $\mathrm{n}^{\circ} 31$, pp. 179-199.

Collet I. (2014), "Les garçons sont-ils des immatures chroniques ? ", Travail, genre et sociétés, $\mathrm{n}^{\circ} 31$, pp. 157-162.

Comaroff J. (1985), Body of Power, Spirit of Resistance. The Culture and History of a South African people, Chicago (III), University of Chicago Press.

Court M. (2010), Corps de filles, corps de garçons : une construction sociale, Paris, La Dispute.

Darmon M. (2006), La socialisation, Paris, Armand Colin.

Denave S. (2019), "'La mécanique, c'est pas son genre ?' Parcours de Sandra en CAP "Maintenance des véhicules automobiles ", in Des jeunes à la marge? Transgressions de sexes et conformité de genre dans les groupes juvéniles, ss. dir. de Buisson-Fenet H. \& Kerivel A., Rennes, PUR.

Depoilly S. (2012), " Des filles conformistes, des garçons déviants. Manières d'être et de faire des élèves des milieux populaires ", Revue française de pédagogie, n 179, pp. 17-28.

Duru-Bellat (2004) (1 ${ }^{\text {ère }}$ éd. 1990), L'école des filles. Quelle formation pour quels rôles sociaux ?, Paris, L'Harmattan.

Goffman E. (1961), Asiles, Études sur la condition sociale des malades mentaux, Éditions de Minuit, Paris.

Grignon J.-C. (1971), L'ordre des choses. Les fonctions sociales de l'enseignement technique, Paris, Éditions de Minuit.

Jellab A. (2001), Scolarité et rapports aux savoirs en lycée professionnel, Paris, PUF.

Jellab A. (2005), «Les enseignants de lycée professionnel et leurs pratiques pédagogiques : entre lutte contre l'échec scolaire et mobilisation des élèves ", Revue française de sociologie, pp. 295-323.

Kergoat P., Jarty J. (2017), «Élèves et enseignants de lycée professionnel. Décryptage d'une relation au prisme des rapports sociaux ", Revue française de pédagogie, n 198, pp. 35-48.

Kergoat P. (2014), «Le travail, l'école et la production des normes de genre. Filles et garçons en apprentissage (en France) », Nouvelles questions féministes, vol. 33, pp. 16-34.

Kergoat P. (2018), De l'indocilité. Apprenti.e.s et élèves de lycées professionnels à l'école et au travail, Habilitation à diriger des recherches, Université de Poitiers. 
Lahire B. (1993), Culture écrite et inégalités scolaires. Sociologie de l'échec scolaire à l'école primaire, Lyon, Presses Universitaires de Lyon.

Lemarchant C. ( 2017), Unique en son genre. Filles et garçons atypiques dans les formations techniques et professionnelles, Paris, PUF.

Lüdtke A. (2015), "La domination comme pratique sociale ", Sociétés contemporaines, $\mathrm{n}^{\circ}$ 99-100, pp. 17-63.

Mardon A., Zeroulou Z. (2015), “'Blédard' et 'fashion victim'. Du bon usage de la mode chez des adolescents d'un quartier populaire ", Hommes et migrations, pp. 101-108.

Marry C. (2014), Les femmes ingénieurs. Une révolution respectueuse, Paris, Belin.

Mathieu N.-C (1971), "Notes pour une définition sociologique des catégories de sexe ", Épistémologie sociologique, 11, pp. 19-39.

Moreau G. (1995), «La mixité dans l'enseignement professionnel », Revue française de pédagogie, $\mathrm{n}^{\circ} 110$, pp. 17-25.

Moreau G. (2006), "École : la double disqualification des lycées professionnels ", in Beaud S., Confavreux J., Lingaard J., La France invisible, Paris, La Découverte.

Mosconi N. (1987), «La mixité dans l'enseignement technique industriel ou l'impossible reconnaissance de l'autre ", Revue française de pédagogie, 78, pp. 31-42

Mosconi N. \& Dahl-Lanotte R. (2003), «C'est technique, est-ce pour elles ? Les filles dans les sections techniques industrielles des lycées ", Travail, genre et sociétés, n 9, pp. 71-90.

Pahleta U. (2012), La domination scolaire. Sociologie de l'enseignement professionnel et de son public, Paris, PUF.

Rubi S. (2005), Les crapuleuses, ces adolescentes déviantes, Paris, PUF.

Rubi S. (2018), Désubstantialiser la question des jeunesses déviantes. Genre, violence et reconnaissance en sociologie de l'éducation, Mémoire d'habilitation à diriger des recherches, Université Paris 8 - Vincennes-Saint-Denis.

Scott J.-C. (2008), La domination et les arts de la résistance. Fragments du discours subalterne, Paris, Éditions Amsterdam.

Skeggs B. (2015), Des femmes respectables. Classe et genre en milieu populaire, Marseille, Agone.

Tabet P. (1979), «Les mains, les outils, les armes », Homme. Revue française d'anthropologie, Tome 19, n' 3-4, pp. 5-61.

Tanguy L. (1983), « Les savoirs enseignés aux ouvriers », Sociologie du travail, nº 3. 